\title{
Seasonal adjustment in a stochastic model
}

\author{
by \\ EKKEHART SCHLICHT
}

Statistische Hefte

1984

number 25

pages 1- 12

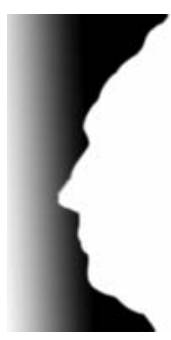

published by

www.semverteilung.vwl.uni-muenchen.de 


\title{
Seasonal adjustment in a stochastic model ${ }^{\star}$
}

\author{
Ekkehart Schlicht
}

O. Introduction

The aim of this paper is to develop a model-based seasonal adjustment method which will yield the same decomposition formulas as the descriptive seasonal adjustment procedures proposed in Schlicht/Pauly [1984] and Schlicht [1981]. Hence the duality between the decriptive and the model-based approaches to seasonal adjustment referred to in Schlicht [1981] is resolved for this class of statistical models and descriptive procedures. In addition, estimates for the weights used in the descriptive procedures can be obtained in the stochastic framework, in principle.

* This paper owes much to many discussions with R. Pauly. He pointed out that the descriptive methods under discussion can be turned into statistical models by introducing a suitable parametrization. He proposed the parametrization by initial values as described in Pauly [1982]; see also Pauly/Schlicht [1984]. This induced the idea of the alternative parametrization developed in this paper.

In contrast to other attempts in this direction notably by Akaike [1980] and Pauly [1982] - the estimation procedure does not involve initial values. Hence the asymmetry in the estimation procedure associated with the introduction of initial values is avoided and a "weakly symmetric" decomposition is obtained [ see Schlicht/Pauly, 1984, section 5.]. On the Schlicht/Pauly procedure and numerical results, see also Astier/Duhamel [1982]. 
1. The Model

Consider a time series $x \in \mathbb{R}^{T}$ which is to be decomposed into a trend $y \in \mathbb{R}^{T}$ and a seasonal component $z \in \mathbb{R}^{T}$. Denote the irregular component by $u \in \mathbb{R}^{T}$, the trend disturbances by $\mathrm{v} \varepsilon \mathbb{R}^{\mathrm{n}}, \mathrm{n}<\mathrm{T}$ and the seasonal perturbations $w \in \mathbb{R}^{\ell}, \ell<\mathrm{T}$. In Schlicht/Pauly [1984] it has been argued that it is reasonable to write

$$
x=y+z+u
$$

$$
P_{Y}=v
$$

$$
\mathrm{Rz}=\mathrm{Zw}
$$

for certain matrices $P, R$, and $Z$. These are assumed to have the following orders and ranks

$\begin{array}{ccccc} & \mathrm{P} & \mathrm{R} & \mathrm{Z} \\ \text { order } & \mathrm{n} \times \mathrm{T} & \mathrm{m} \times \mathrm{T} & \mathrm{m} \times \ell\end{array}$

(4)

rank $n$

with $\mathrm{n}<\mathrm{T}, \mathrm{m}<\mathrm{T}, \mathrm{m}+\mathrm{n} \geq \mathrm{T}, \mathrm{m} \leq \ell \leq \mathrm{T}$

The Schlicht/Pauly adjustment procedure is simply to minimize the expression

$$
u^{\prime} u+\alpha v^{\prime} v+\gamma w^{\prime} w \quad \alpha>0, \gamma>0
$$

for the given time series $\mathrm{x}$ and given weights $\alpha$ and $\gamma$ under the constraints (1) - (3).

This leads to a unique solution

$$
y=y(x ; \alpha, \gamma)
$$

$$
z=z(x ; \alpha, \gamma)
$$


which is defined by

(8) $\left[\begin{array}{ccc}I+\alpha P^{\prime} P & I & y \\ I & I+\gamma R^{\prime}\left(z Z^{\prime}\right)^{-1}{ }_{R} & z\end{array}\right]=$

provided [ $\left.P^{\prime}, R^{\prime}\right]$ is of full rank (which happens to be the case for the specific matrices used in Schlicht/ Pauly [198.4]).

Equations (1)-(3) can be turned into a stochastic model by adding the assumption that the $u, v$, and $w$ are normally distributed disturbances

$$
\mathrm{u} \sim \mathcal{N}\left(0, \sigma_{\mathrm{u}}^{2} \mathrm{I}\right), \mathrm{v} \sim \mathcal{N}\left(0, \sigma_{\mathrm{v}}^{2} \mathrm{I}\right), \mathrm{w} \sim \mathcal{N}\left(0, \sigma_{\mathrm{w}}^{2} \mathrm{I}\right)
$$

Our task will be to show that the maximum likelihood estimates of $y$ and $z$ within the model (1)-(3), (9) are given by (6) and (7) if we put

$$
\alpha=\sigma_{\mathrm{u}}^{2} / \sigma_{\mathrm{v}}^{2}, \gamma=\sigma_{\mathrm{u}}^{2} / \sigma_{\mathrm{w}}^{2}
$$

In order to do that, a further assumption is needed, however:

\section{Assumption 1 (Orthogonality of trend and season):}

11) $\mathrm{Py}=0$ implies Ry $\neq 0$ for all $y \neq 0$.

Remark 1 The specific matrices proposed by Schlicht/ Pauly [1984] satisfy the requirements (4) and (11) if the length of the season $s$ is larger or equal to two and if $T$ is larger than $s$. Condition (4) is satisfied directly. Condition (11) holds true since Py $=0$ implies $y_{t}=a+b t$ for some constants $a$ and $b$, and Ry implies $y_{t}=y_{t-s}$ for all $t$ satisfying $t>s$ and $t \leq T$. In order to achieve both $P y=0$ and $R_{Y}=0, a$ and $b$ need to be zero. 
2. A Parametrization

The first step is to solve (2) und (3) for $y$ and $z$, respectively. Consider first equation (2). A set of $(T-n)$ orthogonal solutions to $\mathrm{PY}=0$ can be normalized and give rise to a matrix $N$ of order $T \times(T-n)$ which satisfies

$$
\mathrm{PN}=0, \quad \mathrm{~N}^{\prime} \mathrm{N}=\mathrm{I}
$$

and any solution to (2) can be written as

$$
y=P^{\prime}\left(P P^{\prime}\right)^{-1} \cdot v+N \cdot \lambda \quad \lambda \in \mathbb{R}^{T-n}
$$

where $\lambda$ stands for a parameter vector.

Similarly there exists a $T \times(T-m)$ matrix M satisfying

$$
R M=0, \quad M \cdot M=I
$$

which permits us to rewrite (3) as

$$
z=R^{\prime}\left(R R^{\prime}\right)^{-1} Z w+M \mu \quad \mu \in \mathbb{R}^{T-m} .
$$

Note that

(16) $\quad \mathrm{N}^{\prime} \mathrm{y}=\lambda, \quad \mathrm{M}^{\prime} \mathrm{z}=\boldsymbol{\mu}$.

Proposition 1 The following holds true

$$
P^{\prime}\left(P^{\prime}\right)^{-1} P+N^{\prime}=I
$$

$$
R^{\prime}\left(R R^{\prime}\right)^{-1} R+M M^{\prime}=I
$$

$$
r(N, M)=T-n+T-m \text {. }
$$

Proof. Statement (17) will be proved first. Consider

$$
\text { (20) }\left[\begin{array}{l}
P \\
N^{\prime}
\end{array}\right] \cdot\left[P^{\prime} N\right] \quad=\left[\begin{array}{ll}
P P^{\prime} & 0 \\
0 & I
\end{array}\right]
$$


which has full rank. Hence the square matrix (P'N)

has full rank. This implies

(2I) $\left[\mathrm{P}^{\prime} \mathrm{N}\right]\left[\begin{array}{cc}(\mathrm{PP})^{-1} & 0 \\ 0 & I\end{array}\right] \cdot\left[\begin{array}{l}\mathrm{P} \\ \mathrm{N}^{\prime}\end{array}\right]=\mathrm{I}$

and hence (17).

Statement (18) is proved analogously.

Statement (19) is implied by assumption 11), qed

3. The Joint Distribution

The system (1), (2), (3), (9) can be replaced now by the equivalent system (1), (13), (14), (9) and we can determine the joint distribubtion of $(x, y, z)$ which is normal

$$
\left(\begin{array}{l}
x \\
y \\
z
\end{array}\right) \sim \mathcal{N}(a, s)
$$

where

$$
a:=\left[\begin{array}{cc}
\mathrm{N} & \mathrm{M} \\
\mathrm{N} & 0 \\
0 & \mathrm{M}
\end{array}\right] \cdot\left[\begin{array}{l}
\lambda \\
\mu
\end{array}\right]
$$

and

$$
s:=\left[\begin{array}{ccc}
s_{x}+s_{y}+s_{z} & s_{y} & s_{z} \\
s_{y} & s_{y} & 0 \\
s_{z} & 0 & s_{z}
\end{array}\right.
$$

with

$$
\begin{aligned}
& S_{x}:=\sigma_{u}^{2} I, S_{y}=\sigma_{v}^{2} P^{\prime}\left(P P^{\prime}\right)^{-1}\left(P^{\prime}\right)^{-1} P \\
& S_{z}:=\sigma_{w}^{2} R^{\prime}\left(R R^{\prime}{ }^{-1}\left(Z^{\prime} Z^{\prime}\right)\left(R R^{\prime}\right)^{-1} R_{R}\right.
\end{aligned}
$$

The parameters are

$$
\theta \quad:=\left(\lambda, \mu, \sigma_{\mathrm{u}}^{2}, \sigma_{\mathrm{v}}^{2}, \sigma_{\mathrm{w}}^{2}\right)
$$


6

Note that $S$ is singular. Hence $\left(x^{\prime}, y^{\prime}, z^{\prime}\right)$ is distributed on a subspace of $\mathbb{R}^{3 T}$ which is determined by the parameters $\lambda$ and $\mu$ : Only those $y$ and $z$ satisfying (16) are possible. Nevertheless we shall speak occasionally about the density of $\left(x^{\prime}, y^{\prime}, z^{\prime}\right)$, and we refer thereby to the density on that subspace.

The distribution (22) gives rise to the marginal distribution

$$
(\mathrm{x} \mid \theta) \sim \mathcal{N}\left(\mathrm{N} \lambda+\mathrm{M \mu}, \mathrm{s}_{\mathrm{x}}+\mathrm{s}_{\mathrm{y}}+\mathrm{s}_{\mathrm{z}}\right)
$$

and the conditional distributions

$$
\left(\begin{array}{l}
y \\
z
\end{array}, \theta\right) \sim \mathcal{N}\left(\left[\begin{array}{ll}
N & 0 \\
0 & M
\end{array}\right]\left[\begin{array}{l}
\lambda \\
\mu
\end{array}\right]+\left[\begin{array}{l}
S_{y} \\
S_{z}
\end{array}\right]\left(S_{x}+S_{y}+S_{z}\right)^{-1}(x-N \lambda-M \mu), S_{y z}\right)
$$

with

29) $S_{y z}:=\left[\begin{array}{ll}S_{y} & \\ 0 & s_{z}\end{array}\right] \quad\left[\begin{array}{l}s_{y} \\ s_{z}\end{array}\right]\left(s_{x}+S_{y}+S_{z}{ }^{-1}\left[s_{y} s_{z}\right]\right.$

(30) $(y \mid x-z, \theta) \sim \mathcal{N}\left(N \lambda+S_{y}\left(S_{x}+S_{y}\right)^{-1}(x-z-N \lambda), S_{y}\left(I-\left(S_{x}+S_{y}{ }^{-1} S_{y}\right)\right)\right.$

(31) $z \mid x-y, \theta) \sim \mathcal{N}\left(M \mu+S_{z}\left(S_{x}+S_{z}{ }^{-1}(x-y-M \mu), S_{z}\left(I-\left(S_{x}+S_{z}{ }^{-1} S_{z}\right)\right.\right.\right.$.

4. Estimates for $\lambda$ and $\mu$

The estimation of the parameters is straightforeward now: We shall derive them as maximum likelihood estimates from (27). Consider first the estimation of $\lambda$ and $\mu$ and assume the variances to be known. Hence the problem boils down to the regression problem described by

$$
\text { 32) } \mathrm{x}=[\mathrm{N}, \mathrm{M}]\left[\begin{array}{l}
\lambda \\
\mu
\end{array}\right]+\varepsilon \text { with } \varepsilon \sim \mathcal{N}\left(0, \mathrm{~S}_{\mathrm{x}}+\mathrm{S}_{\mathrm{Y}}+\mathrm{S}_{\mathrm{z}}\right.
$$


Hence unbiased maximum likelihood estimators are given by the Aitken estimators

33) $\hat{\imath}_{\hat{\mu}}^{\hat{\lambda}}=\left[\left[\begin{array}{l}N \\ M\end{array}\right]\left(S_{x}+S_{y}+S_{z}\right)^{-1}[N, M]\right]^{-1}\left[\begin{array}{l}N \cdot \\ M \cdot\end{array}\right]\left(S_{x}+S_{Y}+S_{z}\right)^{-1} \cdot x$

All matrices involved are of full rank - compare (19)

and (25). Hence the inverses exist. The expression

(33) is equivalent to

34) $\mathrm{N} \cdot\left(\mathrm{S}_{\mathrm{x}}+\mathrm{S}_{\mathrm{y}}+\mathrm{S}_{\mathrm{z}}{ }^{-1}(\mathrm{x}-\mathrm{N} \hat{\mathrm{\lambda}}-\mathrm{M} \hat{\mu}=0\right.$

35) $M^{\prime}\left(S_{x}+S_{y}+S_{z}\right)^{-1}(x-N \hat{\lambda}-M \hat{\mu})=0$

5. Estimates for Trend and Season

If we knew the true parameters $\theta$ (including $\lambda$ and $\mu$ ) we would know from (28) the distribution of trend $y$ and seasonal component $z$, but their "true" values would remain unaccessible. It seems to be reasonable, however, to define the estimates for trend and season by the mode (which equals the expected value) of the distribution (28), since the position of the distribution is characterized by the mode.

Hence we define trend and season as follows

36) $\hat{y}\left(x ; \theta \quad:=N \lambda+S_{y}\left(S_{x}+S_{y}+S_{z}-1-x-N \lambda-M \mu\right)\right.$

37) $\hat{z}\left(x ; \theta \quad:=M \mu+S_{z}\left(S_{x}+S_{y}+S_{z}{ }^{-1}(x-N \lambda-M \mu)\right.\right.$

Now we shall prove the following

Theorem 1 For $\theta=\left(\hat{\lambda}, \hat{\mu}, \sigma_{u}^{2}, \sigma_{v}^{2}, \sigma_{w}^{2}\right)$ and $\hat{\lambda}, \hat{\mu}$ defined in (33) and for $\alpha=\sigma_{\mathrm{u}}^{2} / \sigma_{\mathrm{v}}^{2}$ and $\gamma=\sigma_{\mathrm{u}}^{2} / \sigma_{\mathrm{w}}^{2}$, the descriptive decomposition defined by $(6)-(8)$ and the statistical decomposition (36) - (37) are numerically identical. 
Proof. Equation (8) is equivalent to

(38) $\alpha P^{\prime} P Y=x-y-z$
(39) $\quad \gamma^{\prime}\left(z z^{\prime}\right)^{-1} R z=x-y-z$

where $y$ and $z$ denote the descriptive solution. We shall show that the statistical solution

(40) $\hat{y}=N \hat{\lambda}+S_{y}\left(S_{x}+S_{y}+S_{z}\right)^{-1}(x-N \hat{\lambda}-M \cdot \hat{\mu})$

(41) $\hat{z}=M \hat{\mu}+S_{z}\left(S_{x}+S_{y}+S_{z}\right)^{-1}(x-N \cdot \hat{\lambda}-M \cdot \hat{\mu})$

satisfies equations (28) and (39) for

$\alpha=\sigma_{\mathrm{u}}^{2} / \sigma_{\mathrm{v}}^{2}$ and $\gamma=\sigma_{\mathrm{u}}^{2} / \sigma_{\mathrm{w}}^{2}$. Because all solutions are unique, this will prove the theorem.

Formulas (40) and (4l) imply

(42) $\hat{y}+\hat{z}=N \hat{\lambda}+M_{\mu}+\left(S_{y}+S_{z}\right)\left(S_{x}+S_{y}+S_{z}\right)^{-1}(x-N \hat{\lambda}-M \hat{\mu})$.

From the definition of the covariance matrices (25)

it follows that

(43) $\left(S_{y}+S_{z}\right)\left(S_{x}+S_{y}+S_{z}\right)^{-1}=I-\sigma_{u}^{2}\left(S_{x}+S_{y}+S_{z}\right)^{-1}$.

Hence (42) implies

(44) $x-\hat{y}-\hat{z}=\sigma_{u}^{2}\left(S_{x}+S_{y}+S_{z}\right)^{-1}(x-N \hat{\lambda}-M \cdot \hat{\mu})$.

If we insert this into (40) and (41), it follows that

(45) $\hat{y}=N \hat{\lambda}+\frac{1}{\sigma_{u}^{2}} \cdot S_{y}(x-\hat{y}-\hat{z})$

(46) $\hat{z}=M \hat{\mu}+\frac{1}{\sigma_{u}^{2}} \quad S_{z}(x-\hat{y}-\hat{z})$. 
Premultiplication with $\alpha P^{\prime} P$ and $\gamma R^{\prime}\left(z Z^{\prime}\right)^{-1} R$ yields (47) $\quad \alpha P^{\prime} P \hat{Y}=\alpha \cdot \frac{\sigma_{v}^{2}}{\sigma_{u}^{2}} P^{\prime}\left(P P^{\prime}\right)^{-1} P(x-\hat{y}-\hat{z}$ 48) $\gamma^{\prime}\left(z Z^{\prime}\right)^{-1} R \hat{z}=\gamma \cdot \frac{\sigma_{w}^{2}}{\sigma_{u}^{2}} R^{\prime}\left(R R^{\prime}\right)^{-1} R(x-\hat{y}-\hat{z})$

For $\alpha=\sigma_{\mathrm{u}}^{2} / \sigma_{\mathrm{v}}^{2}$ and $\gamma=\sigma_{\mathrm{u}}^{2} / \sigma_{\mathrm{w}}^{2}$ and with (17 and (18) this can be rewritten as

(49) $\quad \alpha P^{\prime} P \hat{y}=x-\hat{y}-\hat{z}-N^{\prime}(x-\hat{y}-\hat{z}$

(50) $\quad \gamma R^{\prime}\left(z Z^{\prime}\right)^{-1} R \hat{z}=x-\hat{y}-\hat{z}-M M^{\prime}(x-\hat{y}-\hat{z})$

In view of 44 the final terms in $(49$ and $(50$ are

(51) $\mathrm{NN}^{\prime}\left(\mathrm{x}-\hat{\mathrm{y}}-\hat{\mathrm{z}}=\sigma_{\mathrm{u}}^{2} \mathrm{NN} \cdot\left(\mathrm{S}_{\mathrm{x}}+\mathrm{S}_{\mathrm{y}}+\mathrm{S}_{\mathrm{z}}{ }^{-1}(\mathrm{x}-\mathrm{N} \hat{\jmath}-\mathrm{M} \hat{\mu})\right.\right.$

(52) $M^{\prime}\left(x-\hat{y}-\hat{z}=\sigma_{u}^{2} M M^{\prime}\left(S_{x}+S_{y}+S_{z}{ }^{-1}(x-N \hat{\chi}-M \hat{\mu}\right.\right.$

and (34) and (35) imply these terms to be zero. Hence (49) and (50) imply

53) $\alpha P^{\prime} P \hat{y}=x-\hat{y}-\hat{z}$

(54) $\quad \gamma R^{\prime}\left(z^{\prime}\right)^{-1} R \hat{z}=x-\hat{y}-\hat{z}$

which proves the proposition.

6. The Estimation of Variances

In order to estimate $\sigma_{u}^{2}, \sigma_{v}^{2}$, and $\sigma_{w}^{2}$ we put $\lambda=\hat{\lambda}$ and $\mu=\hat{\mu}$ and define the associated errors in $(32$ as

$$
\hat{\varepsilon}=x-N \hat{\imath}-M \hat{\mu} .
$$


In view of Theorem 1 we take furthermore

$$
\begin{aligned}
& \alpha=\sigma_{\mathrm{u}}^{2} / \sigma_{\mathrm{v}}^{2} \\
& \gamma=\sigma_{\mathrm{u}}^{2} / \sigma_{\mathrm{w}}^{2}
\end{aligned}
$$

in the following.

Disregarding constants, the associated concentrated logarithmic likelihood function reads

$$
L\left(\sigma_{u}^{2}, \sigma_{v}^{2}, \sigma_{w}^{2}=l o g \operatorname{det}\left(S_{x}+S_{y}+S_{z}+\hat{\varepsilon} \cdot\left(S_{x}+S_{y}+S_{z}\right)^{-1} \hat{\varepsilon}\right.\right.
$$

last term

59) $A:=\hat{\varepsilon}^{\prime}\left(S_{x}+S_{y}+S_{z}\right)^{-1} \hat{\varepsilon}$

can be simplified further. Define

(60) $\hat{\mathrm{u}}:=\mathrm{x}-\hat{\mathrm{y}}-\hat{\mathrm{z}}, \quad \hat{\mathrm{v}}=\mathrm{P} \hat{\mathrm{Y}}, \quad \hat{\mathrm{w}}=\mathrm{Z}^{\prime}\left(\mathrm{zZ} \mathrm{Z}^{\prime}\right)^{-1} \mathrm{R} \hat{\mathrm{z}}$

as the associated errors resulting from the decomposition defined by ( 8 ). Hence (44) implies

$$
\hat{\mathrm{u}}=\sigma_{\mathrm{u}}^{2}\left(\mathrm{~S}_{\mathrm{x}}+\mathrm{S}_{\mathrm{y}}+\mathrm{S}_{\mathrm{z}}{ }^{-1} \hat{\varepsilon}\right.
$$

and

$$
A=\frac{1}{\sigma_{u}^{4}} \hat{u} \cdot\left(S_{x}+S_{y}+S_{z}\right) \hat{u}
$$

Since (53) and (54) together with the definitions of the covariance matrices imply

$$
\begin{aligned}
& \frac{1}{\sigma_{u}^{4}} \hat{u} \cdot S_{x} \hat{u}=\frac{1}{\sigma_{u}^{2}} \hat{u} \cdot \hat{u} \\
& \frac{1}{\sigma_{u}^{4}} \hat{u} \cdot S_{y} \hat{u}=\frac{1}{\sigma_{v}^{2}} \hat{y}^{\prime} P \cdot P \hat{Y}=\frac{1}{\sigma_{v}^{2}} \hat{v}^{\prime} \hat{v}
\end{aligned}
$$




$$
\frac{1}{\sigma_{u}^{4}} \hat{u}^{\prime} S_{z} \hat{u}=\frac{1}{\sigma_{w}^{2}} \hat{z}^{\prime} R^{\prime}\left(z z^{\prime}\right)^{-1} R \hat{z}=\frac{1}{\sigma_{w}^{2}} \hat{w}^{\prime} \hat{w}
$$

expression A can be written alternatively as

$$
A=\frac{1}{\sigma_{u}^{2}} \hat{u} \cdot \hat{u}+\frac{1}{\sigma_{v}^{2}} \hat{v}^{\prime} \hat{v}+\frac{1}{\sigma_{w}^{2}} \hat{w}^{\prime} \hat{w}
$$

the likelihood function can be written as

(67) $L\left(\sigma_{u}^{2}, \sigma_{v}^{2}, \sigma_{w}^{2}\right)=\log \operatorname{det}\left(S_{x}+S y+S_{z}\right)+\frac{1}{\sigma_{u}^{2}} \hat{u} \cdot \hat{u}+\frac{l}{\sigma_{v}^{2}} \hat{v} \cdot \hat{v}+\frac{1}{\sigma_{w}^{2}} \hat{w}^{\prime} \hat{w}$

and maximum likelihood estimates can be obtained numerically ${ }^{1)}$.

At present P. Duhamel, Université de Paris-Sud, is pursuing this problem in the framework of the Schlicht/Paul procedure via extensive Monte-Carlo studies.

1) From (8) one gets

$$
\text { (*) } \quad A=\frac{1}{\sigma_{u}^{2}}\left(x^{\prime} x-\left[x^{\prime}, x^{\prime}\right] H^{-1}\left[\begin{array}{l}
x \\
x
\end{array}\right]\right)
$$

and

$$
(* *) \frac{\partial A}{\partial \sigma_{u}^{2}}=-\frac{1}{\sigma_{u}^{4}} \hat{u} \cdot \hat{u}, \frac{\partial A}{\partial \sigma_{v}^{2}}=-\frac{I}{\sigma_{v}^{4}} \hat{v} \cdot \hat{v}, \frac{\partial A}{\partial \sigma_{w}^{2}}=-\frac{1}{\sigma_{w}^{4}} \hat{w} \cdot \hat{w}
$$

In addition

$$
(* * *) \quad B=\log \operatorname{det}\left(S_{x}+S_{y}+S_{z}\right)
$$

is homogeneous of degree $T$. This implies the necessary condition

$$
(* * * *) \frac{1}{\sigma_{\mathrm{u}}^{2}} \hat{\mathrm{u}} \cdot \hat{\mathrm{u}}+\frac{1}{\sigma_{\mathrm{v}}^{2}} \hat{\mathrm{v}} \cdot \hat{\mathrm{v}}+\frac{1}{\sigma_{\mathrm{w}}^{2}} \hat{w}^{\prime} \hat{\mathrm{w}}=\mathrm{TB}=\mathrm{A}
$$

for a maximum of (67), but I have been unable to solve the complete problem analytically. 
References

Akaike, H. (1980: Seasonal adjustment by Bayesian modeling. Journal of Time Series Analysis 1,1-13.

Astier, R., Duhamel, C. (1982): Désaisonalisation d'une série chronologique par minimisation des perturbations (mimeo). Centre d'études sur la modélisation et la statistique, Université de Paris-sud.

Pauly, R. (1982): Zerlegung und Analyse ökonomischer Zeitreihen. Statistische Hefte, 291-303.

Pauly, R., Schlicht, E. (1984): Zerlegung ökonomischer Zeitreihen: Ein deterministischer und stochastischer Ansatz. To appear in Allgemeines Statistisches Archiv.

Schlicht, E. (1981): A seasonal adjustment principle and a seasonal adjustment method derived from this principle. Journal of the American Statistical Association 76, 374-378

Schlicht, E., Pauly, R. (1984): Descriptive Seasonal Adjustment by Minimizing Perturbations. To appear in Empirica

Prof.Dr. Ekkehart Schlicht

Technische Hochschule Darmstadt

Fachgebiet Wirtschaftstheorie

Residenzschloß

D-6100 Darmstadt 